\title{
Controller Synthesis to Render a Closed Loop Transfer Function Strongly Strictly Negative Imaginary
}

DOI:

10.1109/cdc.2018.8619279

\section{Document Version}

Accepted author manuscript

Link to publication record in Manchester Research Explorer

\section{Citation for published version (APA):}

Salcan Reyes, G., Lanzon, A., \& Petersen, I. R. (2019). Controller Synthesis to Render a Closed Loop Transfer Function Strongly Strictly Negative Imaginary. In Proceedings of the 57th IEEE Conference on Decision and Control, https://doi.org/10.1109/cdc.2018.8619279

\section{Published in:}

Proceedings of the 57th IEEE Conference on Decision and Control,

\section{Citing this paper}

Please note that where the full-text provided on Manchester Research Explorer is the Author Accepted Manuscript or Proof version this may differ from the final Published version. If citing, it is advised that you check and use the publisher's definitive version.

\section{General rights}

Copyright and moral rights for the publications made accessible in the Research Explorer are retained by the authors and/or other copyright owners and it is a condition of accessing publications that users recognise and abide by the legal requirements associated with these rights.

\section{Takedown policy}

If you believe that this document breaches copyright please refer to the University of Manchester's Takedown Procedures [http://man.ac.uk/04Y6Bo] or contact uml.scholarlycommunications@manchester.ac.uk providing relevant details, so we can investigate your claim.

\section{OPEN ACCESS}




\title{
Controller Synthesis to Render a Closed Loop Transfer Function Strongly Strictly Negative Imaginary
}

\author{
Gabriela Salcan-Reyes, Alexander Lanzon and Ian R. Petersen
}

\begin{abstract}
In this paper, we show that there exists an alternative transformation from the class of negative imaginary to the class of positive real systems. We use this to offer a solution to the problem of designing a controller such that the closed loop is strongly strictly negative imaginary and its associated linear fractional interconnection is internally stable.
\end{abstract}

\section{INTRODUCTION}

Negative imaginary (NI) systems is an important notion within the area of robust control theory because an NI uncertain system can be characterized only by the knowledge of its phase [1] like positive real (PR) systems [2]. Examples of NI engineering applications can be found in [3]. Also, it is known that the negative interconnection of a PR system with a weakly strictly positive real (WSPR) system is internally stable [4], while the positive interconnection of an NI system with a weakly strictly negative imaginary (WSNI) system is internally stable as long as additional gain conditions are satisfied [5]. Then, it is natural to try to relate NI to PR systems. In fact, there exists a transformation from the former to the latter by means of a blocking zero at the origin [1]. However, this transformation has complicated the use of existing solutions of the PR synthesis problem [6], [7], [8], [9], [10], [11] to solve the corresponding NI problem. This is because WSNI systems are transformed into non-strict PR systems and not WSPR systems [1], [12]. For this reason, most of the attempts to solve the NI synthesis problem have produced a nominal closed-loop which is not strictly NI with possible poles on the $j \omega$ axis. Therefore, the internal stability of the associated interconnection is not accomplished in previous literature [12], [13], [14], [15]. Alternatively, [16], [17] offered sufficient conditions based on the solution of two algebraic Riccati equations (ARE) in order to guarantee the closed loop is WSNI and the associated interconnection is internally stable. However, due to the peculiarities of the blocking zero at the origin, one of the involved AREs has a singular Hamiltonian and consequently, its computation

This work was supported by the Engineering and Physical Sciences Research Council (EPSRC) [grant number EP/R008876/1], the Ecuadorian government's Secretaría de Educación Superior, Ciencia, Tecnología e Innovación (Senescyt) and the Australian Research Council under grant D160101121. All research data supporting this publication are directly available within this publication.

G. Salcan-Reyes and A. Lanzon are with the Control Systems Centre, School of Electrical and Electronic Engineering, University of Manchester, United Kingdom Gabriela.Salcan@manchester.ac.uk, Alexander. Lanzon@manchester.ac.uk

Ian R. Petersen is with the research School of Engineering, ANU, Canberra, ACT 260, Australia i.r.petersen@gmail.com

G. Salcan-Reyes is also with Escuela Superior Politécnica del Litoral, ESPOL, FIEC, Campus Gustavo Galindo Km 30.5 Vía Perimetral, P.O. Box 09-01-5863, Guayaquil, Ecuador gapasalceespol .edu.ec

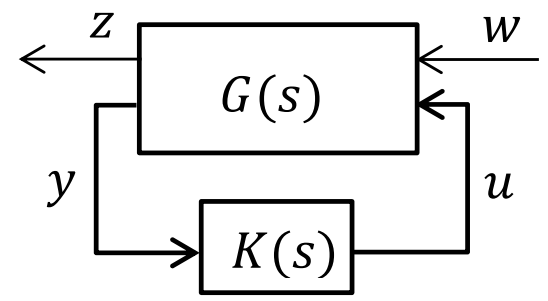

Fig. 1. Standard feedback connection

is numerically challenging. [10] and [11] consider a case which includes performance objectives but the conservative sufficient conditions provided there do not necessarily give a WSNI closed-loop even though they guarantee internal stability of the closed loop. In this paper, we are interested in a WSNI closed-loop such that robust stability is guaranteed against non-strict NI uncertainties such as the spillover dynamics of undamped flexible structures [18]. Furthermore, the conditions we provide are necessary and sufficient.

Notation: Let $\Re(a)$ denote the real part of a complex number $a$. Given two sets $A$ and $B, A \backslash B$ denotes the relative complement of $B$ in $A$, i.e. the set of all elements that belong to $A$ but not to $B$. $I$ stands for an identity matrix of appropriate dimensions. Let $\operatorname{rank}(A), \operatorname{ker} A$ denote the rank and the kernel respectively of a matrix $A$, while $\bar{\lambda}(A)$ denotes the maximum eigenvalue of a matrix $A$ that has only real eigenvalues. $\sigma(A), \bar{\sigma}(A)$ stand for the minimum and maximum singular value of $A$ respectively. We will denote by $\operatorname{herm}(A)$ the Hermitian part of a square matrix, i.e. $\operatorname{herm}(A)=(1 / 2)\left(A+A^{*}\right)$. For a matrix $A \in \mathbb{C}^{n \times m}$ with rank $r, A^{\perp} \in \mathbb{C}^{(n-r) \times n}$ denotes a matrix such that $A^{\perp} A=0$ and $A^{\perp} A^{\perp *}>0 . A^{-T}$ and $G^{\sim}(s)$ are shorthand for $\left(A^{-1}\right)^{T}$ and $G(-s)^{T}$ respectively. For simplicity of notation, we write $F_{\varepsilon}(s)$ instead of $F(s, \varepsilon)$, then $F_{\varepsilon=0}(s)$ represents $F(s, 0)$. A square transfer function $G(s)$ such that $\operatorname{det}(G(s)) \neq 0$ for some $s \in \mathbb{C}$ is said to have full normal rank. $G(s)=\left[\begin{array}{l|l}A & B \\ \hline C & D\end{array}\right]$ denotes the state-space representation of a transfer function $G(s)$. We shall also write the state-space representation of $G(s)$ in a matrix form as $G(s) \stackrel{s}{=} \mathbf{G}=\left[\begin{array}{ll}A & B \\ C & D\end{array}\right] . \mathcal{F}_{l}(G(s), K(s))$ stands for the lower fractional transformation of $G(s)$ and $K(s)$. $\langle G(s), K(s)\rangle$ denotes the lower linear fractional interconnection of Fig. 1. We say $\langle G(s), K(s)\rangle$ is internally stable in the sense of $[19$, Definition A.4.1]. 


\section{PREliminaries}

The following technical results are useful to derive the main results of this paper.

Lemma 1: Let $X, Y, Z$ and $W$ be real matrices of compatible dimensions. Then, $X=X^{T}>0, Y=Y^{T}>0$ and $W=0$ if and only if there exists $\varepsilon_{0}>0$ such that

$$
\left[\begin{array}{cc}
X & W \\
W & 0
\end{array}\right]+\varepsilon\left[\begin{array}{cc}
0 & Z \\
Z^{T} & Y
\end{array}\right]>0 \forall 0<\varepsilon<\varepsilon_{0} .
$$

Proof: $(\Longrightarrow)$ Note that if $X=X^{T}>0$ and $Y=$ $Y^{T}>0$ then

$$
\begin{gathered}
{\left[\begin{array}{cc}
X & \varepsilon Z \\
\varepsilon Z^{T} & \varepsilon Y
\end{array}\right]>0} \\
\Longleftrightarrow \varepsilon>0, Y-\varepsilon Z^{T} X^{-1} Z>0 \\
\Longleftrightarrow \varepsilon>0, \varepsilon \bar{\lambda}\left(Z^{T} X^{-1} Z Y^{-1}\right)<1 .
\end{gathered}
$$

Therefore, if $Z=0$ then (1) holds for all $\varepsilon>0$. On the other hand, when $Z \neq 0$, (1) holds for all $0<\varepsilon<$ $\bar{\lambda}\left(Z^{T} X^{-1} Z Y^{-1}\right)^{-1}$.

( $\Longleftarrow$ ) It is easy to check that $X>0$ and $Y>0$ (since $\varepsilon>0)$. Finally, by continuity

$$
\left[\begin{array}{ll}
X & W \\
W & 0
\end{array}\right] \geq 0
$$

which yields $W=0$.

Lemma 2: Considser a matrix $C_{1} \in \mathbb{C}^{m \times n}$. Let the matrices $X \in \mathbb{C}^{n \times n}, Y \in \mathbb{C}^{n \times n}, S \in \mathbb{C}^{k \times n}, W \in \mathbb{C}^{k \times n}$, $N \in \mathbb{C}^{k \times k}, \tilde{N} \in \mathbb{C}^{k \times k}$ be such that

$$
P=\left[\begin{array}{cc}
N & W \\
W^{T} & X
\end{array}\right]>0 \text { and } P^{-1}=\left[\begin{array}{cc}
\tilde{N} & S \\
S^{T} & Y
\end{array}\right]
$$

and consider vectors $u \in \mathbb{C}^{n}$ and $v \in \mathbb{C}^{m}$. Then, the following two statements are equivalent:

$$
\begin{aligned}
& \text { 1) }\left[\begin{array}{cc}
S & 0 \\
Y & -C_{1}^{T}
\end{array}\right]\left[\begin{array}{l}
u \\
v
\end{array}\right]=0 \text {. } \\
& \text { 2) }\left[\begin{array}{cc}
Y & I_{n} \\
I_{n} & X
\end{array}\right]\left[\begin{array}{cc}
I & 0 \\
0 & -C_{1}^{T}
\end{array}\right]\left[\begin{array}{l}
u \\
v
\end{array}\right]=0 \text {. }
\end{aligned}
$$

Proof: First, note that $Y-X^{-1}=S^{T} \tilde{N}^{-1} S$, then

$$
\begin{aligned}
0 & =\left[\begin{array}{cc}
S & 0 \\
Y & -C_{1}^{T}
\end{array}\right]\left[\begin{array}{l}
u \\
v
\end{array}\right] \\
\Longleftrightarrow 0 & =\left[\begin{array}{cc}
Y-X^{-1} & 0 \\
Y & -C_{1}^{T}
\end{array}\right]\left[\begin{array}{l}
u \\
v
\end{array}\right] \\
\Longleftrightarrow 0 & =\left[\begin{array}{cc}
0 & I \\
-X & X
\end{array}\right]\left[\begin{array}{cc}
Y-X^{-1} & 0 \\
Y & -C_{1}^{T}
\end{array}\right]\left[\begin{array}{l}
u \\
v
\end{array}\right] \\
\Longleftrightarrow 0 & =\left[\begin{array}{ll}
Y & I_{n} \\
I_{n} & X
\end{array}\right]\left[\begin{array}{cc}
I & 0 \\
0 & -C_{1}^{T}
\end{array}\right]\left[\begin{array}{l}
u \\
v
\end{array}\right],
\end{aligned}
$$

which is the desired conclusion.

\section{REVIEW OF PR AND NI SYSTEMS}

We start by recalling the definition of real, rational and proper PR transfer functions. For a detailed discussion on PR systems see [20], [4] and references therein. We remark that in this paper we consider only transfer functions that are real, rational and proper.
Definition 1: A real, rational and proper transfer function matrix $F: \mathbb{C} \rightarrow \mathbb{C}^{m \times m}$ is positive real (PR) if the following conditions are satisfied:

1) $F(s)$ has no poles in $\{s \in \mathbb{C}: \Re(s)>0\}$;

2) $F(j \omega)+F(j \omega)^{*} \geq 0$ for all $\omega \in \mathbb{R}$ except for the values of $\omega$ where $j \omega$ is a pole of $F(s)$;

3) if $s=j \omega_{0}$ with $\omega_{0} \in \mathbb{R}$ is a pole of $F(s)$, then it is a simple pole and the residue matrix of $F(s)$, $K_{0}=\lim _{s \rightarrow j \omega_{0}}\left(s-j \omega_{0}\right) F(s)$, is Hermitian and positive semidefinite.

Next, we recall the weakest notion of strictly PR systems.

Definition 2: A real, rational and proper transfer function $F: \mathbb{C} \rightarrow \mathbb{C}^{m \times m}$ is weakly strictly positive real (WSPR) if

1) $F(s)$ has no poles in $\{s \in \mathbb{C}: \Re(s) \geq 0\}$;

2) $F(j \omega)+F(j \omega)^{*}>0$ for all $\omega \in \mathbb{R}$.

As pointed out in [4] and [21], there are several definitions for the notion of strictly positive real systems and there is no consensus in the literature on this definition. By the definition that we provide below, we propose to contribute with a terminology that is self explanatory and avoids confusion throughout the following sections.

Definition 3: [22] A real, rational and proper transfer function $F: \mathbb{C} \rightarrow \mathbb{C}^{m \times m}$ is strictly positive real with $\gamma$ nullity at $\infty(\operatorname{SPR}(\gamma))$ if

1) $F(s)$ is WSPR;

2) $\lim _{|\omega| \rightarrow \infty} \omega^{2 \gamma} \operatorname{det}\left[F(j \omega)+F(j \omega)^{*}\right] \neq 0$;

where $\gamma$ is the dimension of the null space of $F(\infty)+$ $F(\infty)^{T}$. Furthermore, the above limit in 2) is positive.

Lemma 3: The real, rational and proper transfer function $F: \mathbb{C} \rightarrow \mathbb{C}^{m \times m}$ is

1) $\operatorname{SPR}(0)$ if and only if $F(s)$ is WSPR and $F(\infty)+$ $F(\infty)^{T}>0$

2) $\operatorname{SPR}(m)$ if and only if $F(s)$ is WSPR, $F(\infty)=$ $-F(\infty)^{T}$ and $\lim _{|\omega| \rightarrow \infty} \omega^{2}\left[F(j \omega)+F(j \omega)^{*}\right]>0$.

Some definitions of NI systems are next given below.

Definition 4: [18] A real, rational and proper transfer function matrix $G: \mathbb{C} \rightarrow \mathbb{C}^{m \times m}$ is negative imaginary (NI) if the following conditions are satisfied:

1) $G(s)$ has no poles in $\{s \in \mathbb{C}: \Re(s)>0\}$;

2) $j\left[G(j \omega)-G(j \omega)^{*}\right] \geq 0$ for all $\omega \in(0, \infty)$ except for the values of $\omega$ where $j \omega$ is a pole of $G(s)$;

$3)$ if $s=j \omega_{0}$ with $\omega_{0} \in(0, \infty)$ is a pole of $G(s)$, then it is a simple pole and the residue matrix of $j G(s), K_{0}=$ $\lim _{s \rightarrow j \omega_{0}}\left(s-j \omega_{0}\right) j G(s)$, is Hermitian and positive semidefinite;

4) if $s=0$ is a pole of $G(s)$, then it is at most a double pole and $\lim _{s \rightarrow 0} s^{2} G(s)$ is Hermitian and positive semidefinite.

One important difference between NI and PR systems is that the former allow up to a relative degree of two, while the relative degree of the latter cannot be greater than one.

Next, the weakest notion of strictly NI systems is referred to as WSNI which is the analogous counterpart of WSPR systems.

Definition 5: [1] A real, rational and proper transfer function $G: \mathbb{C} \rightarrow \mathbb{C}^{m \times m}$ is weakly strictly negative imaginary (WSNI) if 
1) $G(s)$ has no poles in $\{s \in \mathbb{C}: \Re(s) \geq 0\}$;

2) $j\left[G(j \omega)-G(j \omega)^{*}\right]>0$ for all $\omega>0$.

Next, we define the analogous counterpart of $\operatorname{SPR}(\gamma)$ systems.

Definition 6: A real, rational and proper transfer function $G: \mathbb{C} \rightarrow \mathbb{C}^{m \times m}$ is strictly negative imaginary with $\gamma$-nullity at $\infty(\mathrm{SNI}(\gamma))$ if

1) $G(s)$ is WSNI;

2) $\lim _{|\omega| \rightarrow \infty} \omega^{2 \gamma+m} \operatorname{det}\left[j\left(G(j \omega)-G(j \omega)^{*}\right)\right] \neq 0$;

where $\gamma=\operatorname{dim} \operatorname{ker}\left\{\lim _{\omega \rightarrow \infty} j \omega\left[G(j \omega)-G(j \omega)^{*}\right]\right\}$. Furthermore, the above limit in 2 ) is positive.

Remark 1: Condition $\lim _{|\omega| \rightarrow \infty} \omega^{2 \gamma+m} \operatorname{det}[j(G(j \omega)-$ $\left.\left.G(j \omega)^{*}\right)\right] \neq 0$ can be easily checked using the state-space realization of $s[G(s)-G(\infty)]$ as in [22, Corollary 1].

We now recall a definition of strongly strictly negative imaginary systems.

Definition 7: [23] A real, rational and proper transfer function $G: \mathbb{C} \rightarrow \mathbb{C}^{m \times m}$ is strongly strictly negative imaginary (SSNI) if

1) $G(s)$ is $\operatorname{SNI}(0)$;

2) $\lim _{\omega \rightarrow 0^{+}} j \frac{1}{\omega}\left[G(j \omega)-G(j \omega)^{*}\right]>0$.

Remark 2: $G(s)$ is $\mathrm{SNI}(0)$ means $G(s)$ is a WSNI system with $\lim _{\omega \rightarrow \infty} j \omega\left[G(j \omega)-G(j \omega)^{*}\right]>0$, i. e. $\operatorname{SNI}(\gamma=0)$.

$\omega \rightarrow \infty$
Remark 3: Note that due to the extra condition 2), SSNI systems are not direct counterparts of $\operatorname{SPR}(0)$ systems.

\section{RELATIONS BETWEEN SNI $(\gamma)$ AND SPR $(\gamma)$ SYSTEMS}

The authors in [21] showed that there exist transformations between $\operatorname{SPR}(\gamma)$ systems and symmetric $\operatorname{SNI}(\gamma)$ systems with a departure condition $\lim _{\omega \rightarrow 0^{+}} j \frac{1}{\omega}\left[G(j \omega)-G(j \omega)^{*}\right]>$ 0 . Those results suggested the possibility of using $\operatorname{SPR}(\gamma)$ controller synthesis methods [6], [7], [24] for the synthesis of $\operatorname{SNI}(\gamma)$ systems. However, the symmetry condition would complicate the direct use of such methods. Here we show that it is possible to extend those results to include nonnecessarily symmetric $\operatorname{SNI}(\gamma)$ transfer functions which do not necessarily satisfy the aforementioned departure condition.

\section{A. $\operatorname{SNI}(\gamma)$ to $\operatorname{SPR}(\gamma)$}

The next theorem shows that a general non-symmetric $\operatorname{SNI}(\gamma)$ system can be transformed into an $\operatorname{SPR}(\gamma)$ system by means of a transmission zero away from the origin.

Theorem 1: Let $G: \mathbb{C} \rightarrow \mathbb{C}^{m \times m}$ be a real, rational, proper $\operatorname{SNI}(\gamma)$ transfer function such that $V^{T} G_{1} V=0$, where $G_{1}=\lim _{\omega \rightarrow \infty} j \omega[G(j \omega)-G(\infty)]$ and the columns of $V \in \mathbb{C}^{m \times \gamma}$ form a basis for the null space of $G_{1}+G_{1}^{T}$. Then, there exists $\varepsilon_{0}>0$ such that $(s+\varepsilon)[G(s)-G(\infty)]$ is $\operatorname{SPR}(\gamma)$ for all $0<\varepsilon<\varepsilon_{0}$.

Proof: Assume, without loss of generality, that $G(s)$ is strictly proper and let $F_{\varepsilon}(s)=(s+\varepsilon) G(s)$. We need to show that for all $0<\varepsilon<\varepsilon_{0}$

1) $F_{\varepsilon}(s)$ has no poles in $\{s \in \mathbb{C}: \Re(s) \geq 0\}$;

2) $F_{\varepsilon}(j \omega)+F_{\varepsilon}(j \omega)^{*}>0$ for all $\omega \in \mathbb{R}$;
3) $\lim _{|\omega| \rightarrow \infty} \omega^{2 \alpha} \operatorname{det}\left[F_{\varepsilon}(j \omega)+F_{\varepsilon}(j \omega)^{*}\right]>0$, where $\alpha=\operatorname{dim} \operatorname{ker}\left\{F_{\varepsilon}(\infty)+F_{\varepsilon}(\infty)^{T}\right\}$;

4) $\alpha=\gamma$.

Note that $F_{\varepsilon}(s)$ and $G(s)$ share the same set of poles with the possible exception of a pole at $s=-\varepsilon$. Therefore condition 1) is satisfied. Also, observe that $F_{\varepsilon}(\infty)=G_{1}$, then condition 4) holds.

Moreover, for each nonzero $\omega \in \mathbb{R}, F_{\varepsilon}(j \omega)+F_{\varepsilon}(j \omega)^{*}>0$ is equivalent to $L_{F_{\varepsilon}}(j \omega)+L_{F_{\varepsilon}}(j \omega)^{*}>0$ by [22, Lemma 4], where

$$
L_{F_{\varepsilon}}(s)= \begin{cases}F_{\varepsilon}(s) & \text { if } \gamma=0 \\
-s^{2} F_{\varepsilon}(s) & \text { if } \gamma=m \\
{\left[\begin{array}{c}
U^{T} \\
-s V^{T}
\end{array}\right] F_{\varepsilon}(s)\left[\begin{array}{ll}
U & s V
\end{array}\right]} & \text { if } 0<\gamma<m\end{cases}
$$

and the columns of $U$ form a basis for the range of $G_{1}+G_{1}^{T}$.

At $\omega=0, F_{\varepsilon}(0)+F_{\varepsilon}(0)^{T}>0$ for every $\varepsilon>0$ since $G(0)=G(0)^{T}>0$ [1]. Then, by continuity of functions there exists a $0<\omega_{1} \in \mathbb{R}$ such that $F_{\varepsilon}(j \omega)+F_{\varepsilon}(j \omega)^{*}>$ $0 \forall|\omega|<\omega_{1}$. Consequently,

$$
L_{F_{\varepsilon}}(j \omega)+L_{F_{\varepsilon}}(j \omega)^{*}>0 \forall 0 \neq|\omega|<\omega_{1} .
$$

In addition, via [22, Corollary 1], if condition 2) is satisfied, condition 3$)$ is equivalent to $\lim _{|\omega| \rightarrow \infty}\left[L_{F_{\varepsilon}}(j \omega)+\right.$ $\left.L_{F_{\varepsilon}}(j \omega)^{*}\right]>0$.

As a result, since $L_{F_{\varepsilon}}$ is continuous, it only remains to show that $L_{F_{\varepsilon}}(j \omega)+L_{F_{\varepsilon}}(j \omega)^{*}$ is nonsingular for all $\omega_{1} \leq$ $|\omega| \in \mathbb{R} \cup\{\infty\}$. This will be done in four steps.

First, rewrite $L_{F_{\varepsilon}}$ as $L_{F_{\varepsilon}}(s)=L_{F}(s)+\varepsilon L_{G}(s)$, where $F(s)=F_{\varepsilon=0}(s)$ and $L_{X}(s)$ is defined as in (3) with $X \in$ $\{F, G\}$ instead of $F_{\varepsilon}$.

Following this, observe that for all $0 \neq \omega \in \mathbb{R}$ we have $F(j \omega)+F(j \omega)^{*}>0$ [23, Lemma 3.1], so $L_{F}(j \omega)+L_{F}(j \omega)^{*}>0$. Also, via [22, Corollary 1] $\lim _{|\omega| \rightarrow \infty} \operatorname{det}\left[L_{F}(j \omega)+L_{F}(j \omega)^{*}\right] \neq 0$ since

$$
\begin{aligned}
0 & \neq \lim _{|\omega| \rightarrow \infty} \omega^{2 \gamma+m} \operatorname{det}\left[j\left(G(j \omega)-G(j \omega)^{*}\right)\right] \\
& =\lim _{|\omega| \rightarrow \infty} \omega^{2 \gamma} \operatorname{det}\left[F(j \omega)+F(j \omega)^{*}\right] .
\end{aligned}
$$

Hence, there exists $\sigma_{1}>0$ such that $\underline{\sigma}\left(L_{F}(j \omega)+\right.$ $\left.L_{F}(j \omega)^{*}\right) \geq \sigma_{1} \forall|\omega| \geq \omega_{1}$.

Next, notice that $G(s) \in \mathcal{R H}_{\infty}, s G(s) \in \mathcal{R} \mathcal{H}_{\infty}$ and $-s^{2} V^{T} G(s) V \in \mathcal{R} \mathcal{H}_{\infty}$ since $G(s)$ is stable, strictly proper and $V^{T} G_{1} V=0$.

Thus, there exists $k_{1}>0$ such that $\bar{\sigma}\left(L_{G}(j \omega)+\right.$ $\left.L_{G}(j \omega)^{*}\right) \leq k_{1}$ for all $|\omega| \geq \omega_{1}$.

Finally, $\underline{\sigma}\left(L_{F_{\varepsilon}}(j \omega)+L_{F_{\varepsilon}}(j \omega)^{*}\right) \geq \sigma_{1}-\varepsilon k_{1}$ for all $|\omega| \geq$ $\omega_{1}$ and by choosing $0<\varepsilon<\varepsilon_{0}=\sigma_{1} / k_{1}$ we guarantee $\operatorname{det}\left[L_{F_{\varepsilon}}(j \omega)+L_{F_{\varepsilon}}(j \omega)^{*}\right] \neq 0$ and therefore conditions 2$)$ and 3$)$ are satisfied, which is the desired conclusion.

Remark 4: Note that if the system $(s+\varepsilon)[G(s)-G(\infty)]$ is $\operatorname{SPR}(\gamma)$ for all $0<\varepsilon<\varepsilon_{0}$, then, by continuity, $s[G(s)-$ $G(\infty)]$ is PR and stable or equivalently $G(s)$ is NI and stable. However, we cannot guarantee that $G(s)$ is $\operatorname{SNI}(\gamma)$.

[11] suggested that any NI transfer function can be transformed into a PR by means of a blocking zero away from 
the origin by choosing a sufficiently small $\varepsilon$. However, the following example shows that transforming an NI into a PR by this transformation is not that simple.

Example 1: Consider the WSNI transfer function $G(s)=$ $\frac{s+3}{(s+1)^{3}} . \quad G(s)$ is not $\operatorname{SNI}(\gamma)$ since $\gamma=1$ and $\lim _{|\omega| \rightarrow \infty} \omega^{3} j\left[G(j \omega)-G(j \omega)^{*}\right]=\lim _{|\omega| \rightarrow \infty} \frac{16 \omega^{4}}{\left(\omega^{2}+1\right)^{3}}=0$. Hence, we cannot guarantee by Theorem 1 that $F_{\varepsilon}(s)=$ $(s+\varepsilon) G(s)$ is $\operatorname{SPR}(\gamma)$ for some $\varepsilon>0$. A simple calculation shows that $F_{\varepsilon}(s)$ is neither $\operatorname{SPR}(\gamma)$ nor WSPR since for all $\varepsilon>0$ there always exists a sufficiently large $\omega$ such that $F_{\varepsilon}(j \omega)+F_{\varepsilon}(j \omega)^{*}=\frac{16 \omega^{2}-2 \varepsilon\left(\omega^{4}+6 \omega^{2}-3\right)}{\left(\omega^{2}+1\right)^{3}}$ is negative.

One might conjecture that the transformation in Theorem 1 suggests the following procedure for an $\operatorname{SNI}(\gamma)$ controller synthesis:

1) Given a generalized plant $G(s)$, find a controller $K(s)$ such that $\mathcal{F}_{l}((s+\varepsilon)(G(s)-G(\infty)), K(s))$ is $\operatorname{SPR}(\gamma)$ and $\langle(s+\varepsilon)(G(s)-G(\infty)), K(s)\rangle$ is internally stable.

2) The obtained controller $K(s)$ will give $\mathcal{F}_{l}(G(s), K(s)$ ) is $\operatorname{SNI}(\gamma)$ and $\langle G(s), K(s)\rangle$ is internally stable.

Unfortunately, as shown in the following example, the conjecture is wrong because it is not guaranteed to obtain an $\operatorname{SNI}(\gamma)$ from an $\operatorname{SPR}(\gamma)$ system by removing the augmented transmission zero. For this reason controller synthesis methods for $\operatorname{SPR}(\gamma)$ systems cannot be directly applied to solve the $\operatorname{SNI}(\gamma)$ synthesis problem as it was suggested by [11].

Example 2: Consider the transfer functions $G(s)$ and $F(s)$ such that $G(s)=\frac{(s+1)}{s^{2}+5 s+6}$ and $F(s)=(s+1) G(s)$. It is easily seen that $F(s)$ is $\operatorname{SPR}(\gamma)$ despite $G(s)$ not being NI since $j\left[G(j \omega)-G(j \omega)^{*}\right]=\frac{2 \omega\left(\omega^{2}-1\right)}{\left(6-\omega^{2}\right)^{2}+25 \omega^{2}}<0$ for all $\omega<1$.

\section{B. SPR(0) and SSNI Lemmas}

It is shown that the SSNI lemma [23] is related to the SPR(0) lemma [7]. These results are of key importance in controller synthesis since we can use the solutions for the SPR(0) synthesis problem [6], [7], [24] in the SSNI synthesis problem. Synthesis is done in the next section.

Theorem 2: Let $G: \mathbb{C} \rightarrow \mathbb{C}^{m \times m}$ be a real, rational, proper transfer function with an arbitrary (i.e. minimal or non-minimal) state-space realization $\left[\begin{array}{l|l}A & B \\ \hline C & D\end{array}\right]$ where $D=$ $D^{T}$. Also, let $F_{\varepsilon}(s)=(s+\varepsilon)(G(s)-D) \stackrel{s}{=} \mathbf{F}_{\varepsilon}$. Then, the following statements are equivalent:

1) There exist an $\varepsilon_{0}>0$ and a real matrix $P=P^{T}>0$ such that herm $\left\{\mathbf{F}_{\varepsilon} \bar{P}\right\}<0$ for all $0<\varepsilon<\varepsilon_{0}$, where $\bar{P}=\left[\begin{array}{cc}P & 0 \\ 0 & -I\end{array}\right]$.

2) $C C^{T}>0$ and there exists a real matrix $P=P^{T}>0$ such that herm $\{A P\}<0, B=-A P C^{T}$.

Furthermore, whenever one of these conditions is satisfied, $F_{\varepsilon}(s)$ is $\operatorname{SPR}(0)$ for all $\varepsilon \in\left(0, \varepsilon_{0}\right)$ (using same $\varepsilon_{0}$ in condition 1)) and $G(s)$ is SSNI.

Proof: Note that $\mathbf{F}_{\varepsilon}=\left[\begin{array}{cc}A & B \\ C(A+\varepsilon I) & C B\end{array}\right]=$
$\left[\begin{array}{l}I \\ C\end{array}\right]\left[\begin{array}{ll}A & B\end{array}\right]+\varepsilon\left[\begin{array}{l}0 \\ I\end{array}\right] C\left[\begin{array}{ll}I & 0\end{array}\right]$, then the relation between 1) and 2) follows by a sequence of equivalent reformulations:

- There exist an $\varepsilon_{0}>0$ and a real matrix $P=P^{T}>0$ such that $\operatorname{herm}\left\{\mathbf{F}_{\varepsilon} \bar{P}\right\}<0$ for all $0<\varepsilon<\varepsilon_{0}$.

- There exist an $\varepsilon_{0}>0$ and a real matrix $P=P^{T}>0$ such that for all $0<\varepsilon<\varepsilon_{0}$,

$$
\left[\begin{array}{cc}
A P+P A^{T} & B+(A-\varepsilon I) P C^{T} \\
B^{T}+C P\left(A^{T}-\varepsilon I\right) & -2 \varepsilon C P C^{T}
\end{array}\right]<0 .
$$

[This equivalence follows on noting that

$$
\begin{aligned}
& {\left[\begin{array}{cc}
I & 0 \\
C & -I
\end{array}\right] 2 \operatorname{herm}\left\{\mathbf{F}_{\varepsilon} \bar{P}\right\}\left[\begin{array}{cc}
I & C^{T} \\
0 & -I
\end{array}\right]} \\
& \left.=\left[\begin{array}{cc}
A P+P A^{T} & B+(A-\varepsilon I) P C^{T} \\
B^{T}+C P\left(A^{T}-\varepsilon I\right) & -2 \varepsilon C P C^{T}
\end{array}\right] .\right]
\end{aligned}
$$

- There exists a real matrix $P=P^{T}>0$ such that $\operatorname{herm}\{A P\}<0, B+A P C^{T}=0$ and $C$ is full row rank.

[This equivalence follows by Lemma 1.]

Furthermore, $F_{\varepsilon}(s)$ is $\operatorname{SPR}(0)$ by 1$)$ and the $\operatorname{SPR}(0)$ lemma [7], while $G(s)$ is SSNI by 2) and [23, Theorem 3.3].

Remark 5: Note that while condition 1) implies that $F_{\varepsilon}(s)$ is $\operatorname{SPR}(0)$ for all $0<\varepsilon<\varepsilon_{0}$, the converse does not necessarily hold since $P$ may not be constant for every $\varepsilon \in\left(0, \varepsilon_{0}\right)$.

\section{SSNI SYNTHESIS PROBLEM}

Consider the uncertain linear system depicted in Fig. 1. The plant $G: \mathbb{C} \rightarrow \mathbb{C}^{(m+p) \times(m+q)}$ is described by

$$
\left[\begin{array}{c}
\dot{x} \\
z \\
y
\end{array}\right]=\left[\begin{array}{ccc}
A & B_{1} & B_{2} \\
C_{1} & D_{11} & D_{12} \\
C_{2} & D_{21} & D_{22}
\end{array}\right]\left[\begin{array}{l}
x \\
w \\
u
\end{array}\right]
$$

where $x$ is the state of the plant, $w$ is the disturbance acting on the system, $u$ is the control input, $z$ is the controlled output signal, $y$ is the measurement output, $A \in \mathbb{R}^{n \times n}$, $B_{1} \in \mathbb{R}^{n \times m} \quad B_{2} \in \mathbb{R}^{n \times q}, C_{1} \in \mathbb{R}^{m \times n}, C_{2} \in \mathbb{R}^{p \times n}$, $D_{11} \in \mathbb{R}^{m \times m}, D_{12} \in \mathbb{R}^{m \times q}, D_{21} \in \mathbb{R}^{p \times m}, D_{22} \in \mathbb{R}^{p \times q}$, and $q \leq m, p \leq m$. We will assume $D_{22}=0$ without loss of generality.

The controller $K: \mathbb{C} \rightarrow \mathbb{C}^{q \times p}$ is described by

$$
\left[\begin{array}{c}
\dot{x}_{k} \\
u
\end{array}\right]=\left[\begin{array}{cc}
A_{k} & B_{k} \\
C_{k} & D_{k}
\end{array}\right]\left[\begin{array}{c}
x_{k} \\
y
\end{array}\right]
$$

where $x_{k}$ is the state of the controller, $A_{k} \in \mathbb{R}^{k \times k}, B_{k} \in$ $\mathbb{R}^{k \times p}, C_{k} \in \mathbb{R}^{q \times k}$ and $D_{k} \in \mathbb{R}^{q \times p}$.

The closed-loop system $\mathcal{F}_{l}(G(s), K(s))$ can be described by

$$
\left[\begin{array}{c}
\dot{x}_{c} \\
z
\end{array}\right]=\left[\begin{array}{ll}
A_{c} & B_{c} \\
C_{c} & D_{c}
\end{array}\right]\left[\begin{array}{c}
x_{c} \\
w
\end{array}\right]
$$


where $x_{c}=\left[\begin{array}{ll}x^{T} & x_{k}^{T}\end{array}\right]^{T}$ is the closed-loop state and

$$
\begin{aligned}
& {\left[\begin{array}{ll}
A_{c} & B_{c} \\
C_{c} & D_{c}
\end{array}\right]=\left[\begin{array}{ll}
Q & R \\
Z & F
\end{array}\right]+\left[\begin{array}{l}
J \\
H
\end{array}\right] \mathbf{K}\left[\begin{array}{ll}
M & E
\end{array}\right]} \\
& {\left[\begin{array}{lll}
Q & R & J \\
Z & F & H \\
M & E & \mathbf{K}
\end{array}\right]=\left[\begin{array}{cc:c:cc}
0_{k} & 0 & 0 & I_{k} & 0 \\
0 & A & B_{1} & 0 & B_{2} \\
\hdashline 0 & C_{1} & D_{11} & 0 & \bar{D}_{12} \\
\hdashline I_{k} & 0 & 0 & A_{k} & B_{k} \\
0 & C_{2} & D_{21} & C_{k} & D_{k}
\end{array}\right] .}
\end{aligned}
$$

Our aim is to find conditions for the existence of an output feedback controller $K(s)$ such that the closed-loop system $\mathcal{F}_{l}(G(s), K(s))$ is SSNI and $\langle G(s), K(s)\rangle$ is internally stable. When such a controller exists, we should provide a way to construct it.

Theorem 3 ( SSNI dynamic output feedback synthesis): Consider the system $G$ in (4) and assume $\left(A, B_{2}\right)$ is stabilizable, $\left(C_{2}, A\right)$ is detectable, $D_{12}=0$ and $D_{22}=0$. Let $U$ and $V$ be matrices such that the columns of $U$ form a basis of $\operatorname{ker}\left[\begin{array}{ll}C_{2} & D_{21}\end{array}\right] \backslash \operatorname{ker}\left[\begin{array}{ll}A & B_{1}\end{array}\right]$ while the columns of $V$ form a basis of $\operatorname{ker}\left[\begin{array}{cc}A & B_{1} \\ C_{2} & D_{21}\end{array}\right]$. Then, there exist a controller $K(s)$ of order $k$ and a real matrix $P=P^{T}>0$ such that $\mathcal{F}_{l}(G(s), K(s))$, as defined in (6) to (8), satisfies $C_{c} C_{c}^{T}>0$,

$$
\operatorname{herm}\left\{A_{c} P\right\}<0 \text { and } B_{c}=-A_{c} P C_{c}^{T}
$$

if and only if there exist real matrices $X=X^{T}>0$ and $Y=Y^{T}>0$ satisfying all of the following conditions:

1) $C_{1} C_{1}^{T}>0$

2) when $\operatorname{det}\left(B_{2} B_{2}^{T}\right)=0$, herm $\left\{B_{2}^{\perp} A X B_{2}^{\perp T}\right\}<0$ and $B_{2}^{\perp}\left(B_{1}+A X C_{1}^{T}\right)=0 ;$

3) herm $\left\{U^{T}\left[\begin{array}{c}Y \\ -C_{1}\end{array}\right]\left[\begin{array}{ll}A & B_{1}\end{array}\right] U\right\}<0$;

4) $\left[\begin{array}{cc}Y & I_{n} \\ I_{n} & X\end{array}\right]\left[\begin{array}{cc}I & 0 \\ 0 & -C_{1}^{T}\end{array}\right] V=0$;

5) $\left[\begin{array}{ll}Y & I_{n} \\ I_{n} & X\end{array}\right] \geq 0$; and

6) $\operatorname{rank}\left(Y-X^{-1}\right) \leq k$.

Furthermore, when these conditions hold, such a controller $K(s)$ can be constructed by solving the linear matrix inequalities (LMI) conditions (9) for the controller state-space matrices $\mathbf{K}$ on using $P=\left[\begin{array}{cc}N & W \\ W^{T} & X\end{array}\right]$ wherein $W$ and $N$ are matrices that satisfy $X-Y^{-1}=W^{T} N^{-1} W$. If, in addition $D_{11}=D_{11}^{T}$, then $\mathcal{F}_{l}(G(s), K(s))$ is SSNI and $\langle G(s), K(s)\rangle$ is internally stable.

Proof: This is a proof sketch. A more detailed proof will be published in a journal publication. Let $U=\left[\begin{array}{ll}U_{1}^{T} & U_{2}^{T}\end{array}\right]^{T}$ and $V=\left[\begin{array}{ll}V_{1}^{T} & V_{2}^{T}\end{array}\right]^{T}$. Let $L=$ - herm $\left\{A_{c} P\right\}$ in (9). Note that $C_{c}=Z$ and that (9) can be rewritten as

$$
\begin{aligned}
-\left[\begin{array}{ll}
L & 0 \\
0 & 0
\end{array}\right] & =\operatorname{herm}\left\{\left[\begin{array}{l}
I \\
0
\end{array}\right]\left[\begin{array}{ll}
A_{c} & B_{c}
\end{array}\right]\left[\begin{array}{cc}
P & P C_{c}^{T} \\
0 & I
\end{array}\right]\right\} \\
& =\operatorname{herm}(\Gamma \mathbf{K} \Lambda+\Theta),
\end{aligned}
$$

where

$$
\left[\begin{array}{ccc}
\Gamma & \Lambda^{T} & \Theta
\end{array}\right]=\left[\begin{array}{c|c|cc}
J & P M^{T} & Q P & Q P Z^{T}+R \\
0 & Z P M^{T}+E^{T} & 0 & 0
\end{array}\right] .
$$

We will prove the statements in this theorem by a sequence of equivalent reformulations:

- $Z Z^{T}>0$, there exists real matrices $P=P^{T}>0$, $L=L^{T}>0$ and a controller $K(s)$ such that

$$
\operatorname{herm}\{\Gamma \mathbf{K} \Lambda+\Theta\}=-\left[\begin{array}{ll}
L & 0 \\
0 & 0
\end{array}\right] .
$$

- There exist a real matrix $P=P^{T}>0$, a controller $K(s)$ and an $\varepsilon_{0}>0$ such that fo all $0<\varepsilon<\varepsilon_{0}$

$$
\operatorname{herm}\{\Gamma \mathbf{K} \Lambda+\Theta\}-\frac{\varepsilon}{2}\left[\begin{array}{cc}
0 & P Z^{T} \\
Z P & 2 Z P Z^{T}
\end{array}\right]<0 .
$$

[By Lemma 1]

- There exist a real matrix $P=P^{T}>0$ and an $\varepsilon_{0}>0$ such that for all $0<\varepsilon<\varepsilon_{0}$ the following two conditions hold:

$$
\begin{array}{r}
\operatorname{herm}\left\{\Gamma^{\perp}\left\{\Theta-\varepsilon\left[\begin{array}{cc}
0 & 0 \\
Z P & Z P Z^{T}
\end{array}\right]\right\} \Gamma^{\perp T}\right\}<0, \\
\operatorname{herm}\left\{\Lambda^{T \perp}\left\{\Theta-\varepsilon\left[\begin{array}{cc}
0 & 0 \\
Z P & Z P Z^{T}
\end{array}\right]\right\} \Lambda^{T \perp T}\right\}<0 .
\end{array}
$$

[By [25], [26]]

- There exist a real matrix $P=\left[\begin{array}{cc}N & W \\ W^{T} & X\end{array}\right]>0$ with $P^{-1}=\left[\begin{array}{cc}\tilde{N} & S \\ S^{T} & Y\end{array}\right]$ and an $\varepsilon_{0}>0$ such that for all $0<\varepsilon<\varepsilon_{0}$ the following two conditions hold:

$$
\begin{gathered}
\text { herm }\left\{\left[\begin{array}{ll}
B_{2}^{\perp} A X B_{2}^{\perp T} & B_{2}^{\perp}\left(A X C_{1}^{T}+B_{1}\right) \\
-\varepsilon C_{1} X B_{2}^{\perp T} & -\varepsilon C_{1} X C_{1}^{T}
\end{array}\right]\right\}<0, \\
\operatorname{herm}\left\{\left[\begin{array}{l}
U^{T} \\
V^{T}
\end{array}\right]\left[\begin{array}{cc}
Y A & Y B_{1} \\
-C_{1}(A+\varepsilon I) & -C_{1} B_{1}
\end{array}\right]\left[\begin{array}{l}
U^{T} \\
V^{T}
\end{array}\right]\right\}<0 .
\end{gathered}
$$

[The equivalence follows by simple substitution and also on noting that

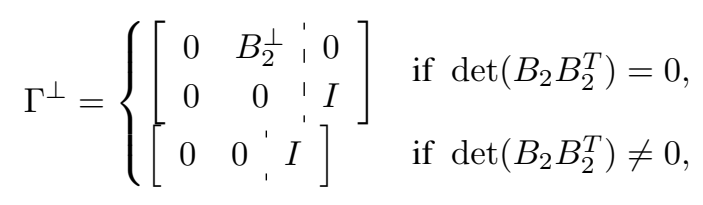

$\Lambda^{T \perp}=\left[\begin{array}{c}M^{T} \\ E^{T}\end{array}\right]^{\perp}\left[\begin{array}{cc}P^{-1} & 0 \\ -Z & I\end{array}\right]$ and the columns of $U$ together with the columns of $V$ form a basis of $\operatorname{ker}\left[\begin{array}{ll}C_{2} & D_{21}\end{array}\right]$ which yields $\left[\begin{array}{c}M^{T} \\ E^{T}\end{array}\right]^{\perp T}=$ $\left.\left[\begin{array}{cc}0 & 0 \\ U_{1} & V_{1} \\ \hdashline U_{2}^{-} & -V_{2}\end{array}\right].\right]$

- There exist $X>0$ and $Y>0$ such that the six conditions of Theorem 2 hold.

[Conditions 5) and 6) follow by [27, Lemma 7.5], while conditions 1) and 2) via Lemma 1. Furthermore, conditions 3) and 4) follow by Lemma 1, Lemma 2 and some algebraic manipulations, but because of space 
limitation, details are not provided here although they will be published elsewhere.]

The final part of the proof follows from Theorem 2 on also noting that $A_{c}$ is Hurwitz via (9) which gives an internally stable $\langle G(s), K(s)\rangle$.

Remark 6: When $\operatorname{rank}(U)=0$, the associated condition should be ignored. The same applies when $\operatorname{rank}(V)=0$.

Remark 7: The previous theorem solves the SSNI problem under some mild technical assumptions on $D_{12}$ and $D_{22}$. These assumptions can be easily circumvented as shown in [28, Lemma 5].

\section{CONCLUSIONS}

We have derived necessary and sufficient conditions for the solution of SSNI synthesis problem. To achieve this, we showed that there exists a transformation from SSNI to $\operatorname{SPR}(0)$ systems and a relation between their lemmas. The solution to the SSNI synthesis problem is based on LMIs conditions which do not impose any restriction in the uncertainty but some mild technical assumptions on the generalized plant which can be easily circumvented. The results of this paper have immediate applications in robust control such as in the control of undamped flexible structures [18], [29], which we are currently investigating.

\section{REFERENCES}

[1] A. Lanzon and I. R. Petersen, "Stability robustness of a feedback interconnection of systems with negative imaginary frequency response," IEEE Transactions on Automatic Control, vol. 53, no. 4, pp. 10421046, May 2008.

[2] W. M. Haddad and D. S. Bernstein, "Robust stabilization with positive real uncertainty: Beyond the small gain theorem," Systems and Control Letters, vol. 17, no. 3, pp. 191-208, Sep 1991.

[3] I. R. Petersen, "Negative imaginary systems theory and applications," Annual Reviews in Control, vol. 42, pp. 309-318, 2016.

[4] B. Brogliato, R. Lozano, B. Maschke, and O. Egeland, Dissipative systems analysis and control: Theory and Applications, 2nd ed. London, UK: Springer-Verlag London, 2007.

[5] A. Lanzon and H. J. Chen, "Feedback stability of negative imaginary systems," IEEE Transactions on Automatic Control, vol. 62, pp. 56205633, Nov 2017.

[6] W. Sun, P. P. Khargonekar, and D. Shim, "Solution to the positive real control problem for linear time-invariant systems," IEEE Transactions on Automatic Control, vol. 39, no. 10, pp. 2034-2046, Oct 1994.

[7] L. Turan, M. Safonov, and C. Huang, "Synthesis of positive real feedback systems: a simple derivation via Parrott's theorem," IEEE Transactions on Automatic Control, vol. 42, no. 8, pp. 1154-1157, 1997.

[8] C. Scherer, P. Gahinet, and M. Chilali, "Multiobjective outputfeedback control via LMI optimization," IEEE Transactions on Automatic Control, vol. 42, no. 7, pp. 896-911, Jul 1997.

[9] X. Li, H. Gao, and C. Wang, "Generalized kalmanyakubovichpopov lemma for 2-d fm lss model," IEEE Transactions on Automatic Control, vol. 57, no. 12, pp. 3090-3103, Dec 2012.

[10] K. Z. Liu, M. Ono, X. Li, and M. Wu, "Robust performance synthesis for systems with positive-real uncertainty and an extension to the negative-imaginary case," Automatica, vol. 82, pp. 194-201, Aug 2017.

[11] K. Z. Liu, M. Ono, X. Huo, and M. Wu, "Robust performance design for systems with output strictly-passive uncertainty," IEEE Transactions on Industrial Electronics, vol. 65, no. 5, pp. 4207-4215, May 2018.

[12] Z. Song, A. Lanzon, S. Patra, and I. R. Petersen, "A negativeimaginary lemma without minimality assumptions and robust statefeedback synthesis for uncertain negative-imaginary systems," Systems \& Control Letters, vol. 61, pp. 1269-1276, Dec 2012.
[13] I. R. Petersen and A. Lanzon, "Feedback control of negative-imaginary systems," IEEE Control Systems Magazine, vol. 30, no. 5, pp. 54-72, Oct 2010.

[14] M. Mabrok, A. G. Kallapur, I. R. Petersen, and A. Lanzon, "A generalized negative imaginary lemma and Riccati-based static statefeedback negative imaginary synthesis," Systems \& Control Letters, vol. 77, pp. 63-68, Mar 2015.

[15] J. Xiong, J. Lam, and I. R. Petersen, "Output feedback negative imaginary synthesis under structural constraints," Automatica, vol. 71, pp. 222-228, Sep 2016.

[16] G. Salcan-Reyes and A. Lanzon, "Negative imaginary synthesis via dynamic output feedback and static state feedback: A Riccati approach," Submitted for publication.

[17] — - "On negative imaginary synthesis via solutions to Riccati equations," in Proceedings of the 16th European Control Conference, Limassol, Cyprus, June 2018, pp. 870-875.

[18] M. A. Mabrok, A. G. Kallapur, I. R. Petersen, and A. Lanzon, "Generalizing negative imaginary systems theory to include free body dynamics: Control of highly resonant structures with free body motion," IEEE Transactions on Automatic Control, vol. 59, no. 10, pp. 2692-2707, Oct 2014.

[19] M. Green and D. J. N. Limebeer, Linear robust control. Englewood Cliffs, NJ, USA: Prentice Hall, 1995.

[20] B. D. O. Anderson and S. Vongpanitlerd, Network Analysis and Synthesis: A Modern Systems Theory Approach. Englewood Cliffs, NJ, USA: Prentice Hall, 1973.

[21] A. Ferrante, A. Lanzon, and L. Ntogramatzidis, "Foundations of not necessarily rational negative imaginary systems theory: Relations between classes of negative imaginary and positive real systems," IEEE Transactions on Automatic Control, vol. 61, no. 10, pp. 3052-3057, Oct 2016

[22] M. Corless and R. Shorten, "On the characterization of strict positive realness for general matrix transfer functions," IEEE Transactions on Automatic Control, vol. 55, no. 8, pp. 1899-1904, 2010.

[23] A. Lanzon, Z. Song, S. Patra, and I. R. Petersen, "A strongly strict negative-imaginary lemma for non-minimal linear systems," Соттиnications in Information and Systems, vol. 11, no. 2, pp. 139-152, 2011.

[24] R. E. Skelton and T. Iwasaki, "Increased roles of linear algebra in control education," IEEE Control Systems, vol. 15, no. 4, pp. 76-90, Aug 1995.

[25] P. Gahinet and P. Apkarian, "A linear matrix inequality approach to $\mathcal{H}_{\infty}$ control," International journal of robust and nonlinear control, vol. 4, pp. 421-448, 1994.

[26] T. Iwasaki and R. E. Skelton, "All controllers for the general $\mathcal{H}_{\infty}$ control problem: LMI existence conditions and state space formulas," Automatica, vol. 30, no. 8, pp. 1307-1317, 1994.

[27] A. Packard, K. Zhou, P. Pandey, and G. Becker, "A collection of robust control problems leading to LMI's," in Proceedings of the 30th IEEE Conference on Decision and Control, Brighton, England, 1991, pp. $1245-1250$.

[28] Z. Song, A. Lanzon, S. Patra, and I. R. Petersen, "Towards controller synthesis for systems with negative imaginary frequency response," IEEE Transactions on Automatic Control, vol. 55, no. 6, pp. 15061511, Jun 2010.

[29] R. Bastaits, G. Rodrigues, B. Mokrani, and A. Preumont, "Active Optics of Large Segmented Mirrors: Dynamics and Control," Journal of Guidance, Control, and Dynamics, vol. 32, no. 6, pp. 1795-1803, Nov-Dec 2009. 